\title{
BMJ Global Health A cluster randomised trial of cookstove interventions to improve infant health in Ghana
}

Darby W Jack (D) , ${ }^{1}$ Kenneth Ayuurebobi Ae-Ngibise, ${ }^{2}$ Carlos F Gould, ${ }^{1}$ Ellen Boamah-Kaali, ${ }^{2}$ Alison G Lee, ${ }^{3}$ Mohammed Nuhu Mujtaba, ${ }^{2}$ Steven Chillrud, ${ }^{4}$ Seyram Kaali, ${ }^{2}$ Ashlinn K Quinn (D) , ${ }^{5}$ Stephaney Gyaase, ${ }^{2}$ Felix Boakye Oppong, ${ }^{2}$ Daniel Carrión, ${ }^{6}$ Oscar Agyei, ${ }^{2}$ Katrin Burkhart, ${ }^{7}$ Joseph A Ana-aro, ${ }^{2}$ Xinhua Liu, ${ }^{8}$ Yvonne Afrah Berko, ${ }^{2}$ Blair J Wylie, ${ }^{9}$ Seeba Amenga Etego, ${ }^{2}$ Robin Whyatt, ${ }^{1}$ Seth Owusu-Agyei, ${ }^{10}$ Patrick Kinney, ${ }^{11}$ Kwaku Poku Asante ${ }^{2}$

To cite: Jack DW,

Ae-Ngibise KA, Gould CF, et al. A cluster randomised trial of cookstove interventions to improve infant health in Ghana. BMJ Global Health 2021;6:e005599. doi:10.1136/ bmjgh-2021-005599

Handling editor Seye Abimbola

- Additional supplemental material is published online only. To view, please visit the journal online (http://dx.doi.org/10. 1136/bmjgh-2021-005599).

Received 5 March 2021 Accepted 24 June 2021

Check for updates

C Author(s) (or their employer(s)) 2021. Re-use permitted under CC BY-NC. No commercial re-use. See rights and permissions. Published by BMJ.

For numbered affiliations see end of article.

Correspondence to Mr Darby W Jack dj2183@cumc.columbia.edu

\section{ABSTRACT}

Introduction Household air pollution from solid fuel combustion for cooking and heating is a leading cause of childhood morbidity and mortality worldwide. We hypothesised that clean cooking interventions delivered during pregnancy would improve child health.

Methods We conducted a cluster randomised trial in rural Ghana to test whether providing pregnant women liquefied petroleum gas (LPG) cookstoves or improved biomass cookstoves would reduce personal carbon monoxide and fine particulate pollution exposure, increase birth weight and reduce physician-assessed severe pneumonia in the first 12 months of life, compared with control participants who continued to cook with traditional stoves. Primary analyses were intention-to-treat. The trial was registered with ClinicalTrials.gov and follow-up is complete.

Results Enrolment began on 14 April 2014, and ended on 20 August 2015. We enrolled 1414 pregnant women; 361 in the LPG arm, 527 in the improved biomass cookstove arm and 526 controls. We saw no improvement in birth weight (the difference in mean birth weight for LPG arm births was $29 \mathrm{~g}$ lighter (95\% $\mathrm{Cl}-113$ to $56, \mathrm{p}=0.51$ ) and for improved biomass arm births was $9 \mathrm{~g}$ heavier $(95 \% \mathrm{Cl}-64$ to $82, \mathrm{p}=0.81)$, compared with control newborns) nor severe child pneumonia (the rate ratio for pneumonia in the LPG arm was 0.98 (95\% Cl 0.58 to $1.70 ; p=0.95)$ and for the improved biomass arm was $1.21(95 \% \mathrm{Cl} 0.78$ to $1.90 ; p=0.52)$, compared with the control arm). Air pollution exposures in the LPG arm remained above WHO health-based targets (LPG median particulate matter less than 2.5 microns in diameter $\left(\mathrm{PM}_{2.5}\right) 45 \mu \mathrm{g} /$ $\mathrm{m}^{3}$; IQR 32-65 vs control median $\mathrm{PM}_{25} 67 \mu \mathrm{g} / \mathrm{m}^{3}$, IQR 46-97).

Conclusions Neither prenatally-introduced LPG nor improved biomass cookstoves improved birth weight or reduced severe pneumonia risk in the first 12 months of life. We hypothesise that this is due to lower-than-expected exposure reductions in the intervention arms. Trial registration number NCT01335490.

\section{Key questions}

What is already known?

- Air pollution exposure arising from cooking with biomass fuels is a major driver of disease and mortality in low-income and middle-income countries (LMICs), and better evidence is needed to guide and to justify investments in clean household energy transitions.

- Previous randomised controlled cookstove trials in Guatemala, Malawi, Peru, Nepal, Nigeria and Rwanda, have yielded null results or shown relatively small health benefits.

- The notable exception to these small effects is the RESPIRE trial in Guatemala, which found that a chimney stove intervention resulted in a rate ratio of $0.67(95 \% \mathrm{Cl}$ 0.45 to 0.98 ) for severe physician diagnosed pneumonia.

\section{What are the new findings?}

- In a cluster randomised controlled trial, we tested the ability of clean stoves and fuels to reduce household air pollution exposures and to improve child health.

- Our study enrolled pregnant women into three arms: a liquefied petroleum gas (LPG) arm (which provided both stoves and monthly free fuel deliveries), an improved biomass stove arm and a control arm.

- In both intention-to-treat and in adjusted analyses, we found no evidence of benefit from either of our interventions, though the LPG arm did experience a reduction in personal air pollution exposures.

What do the new findings imply?

- We contribute to a growing body of evidence from randomised controlled trials that suggests that household-level interventions are unlikely to fully address health risks from polluting cookstoves.

- Household energy policy in LMICs should focus on addressing all sources of air pollution at the community level if it is to generate the air pollution exposure reductions necessary to improve health.

- This will require both clean and affordable household energy for all, and also strategies to address emissions from traffic, trash burning and other sources. 


\section{INTRODUCTION}

In 2019, air pollution caused an estimated 6.7 million premature deaths. ${ }^{1}$ Household air pollution (HAP) from incomplete combustion of solid fuels (wood, dung, crop residue and charcoal) in inefficient home cookstoves in low-income and middle-income countries (LMICs) accounts for about one-third of this mortality burden, and in some areas is also an important source of ambient air pollution. Systematic reviews have found associations between HAP exposure and morbidity and mortality across the lifecourse, including reductions in birth weight ${ }^{2}$ and increased risk for early childhood acute lower respiratory illnesses (ALRI), the leading killer of children under 5 years of age. ${ }^{3}$ In Ghana, approximately 11000 deaths and 467000 disability adjusted life years annually are attributed to HAP exposure. Despite this burden, evidence regarding the efficacy of a cookstove intervention to reduce HAP exposure and improve health outcomes is sparse. Previous randomised controlled cookstove trials in Guatemala, ${ }^{4}$ Malawi, ${ }^{5}$ Peru, ${ }^{6} \mathrm{Nepal}^{7}$ and Rwanda, ${ }^{8}$ have yielded null results or shown small health benefits (with the exception of severe pneumonia in the RESPIRE trial in Guatemala, which showed meaningful reductions in severe pneumonia). These trials assessed improved biomass-burning cookstoves and, with the exception of the Nepal trial, did not study the use of clean fuels.

We conducted a cluster-randomised controlled trial in which households with a pregnant woman as primary cook were randomised to receive a liquefied petroleum gas (LPG) cookstove, the cleanest household fuel available at scale in LMICs, ${ }^{8}$ a low-cost, high-efficiency biomass cookstove that allows burning of locally-available fuels or no cookstove intervention. We refer to this study as the Ghana Randomized Air Pollution and Health Study (GRAPHS). We hypothesised that these cookstove interventions would increase birth weight and decrease risk of physician-assessed severe pneumonia in the first 12 months of life. These outcomes were selected both because they are associated with a significant global burden of disease, ${ }^{1}$ can influence the course of health outcomes over the lifespan and because we believed that cookstove interventions that target pregnant women could, if effective, be deployed through established antenatal care systems. The study was jointly developed by a research team from the USA and from Ghana. Ghana was an ideal place for this research because solid fuel use was-and remains-ubiquitous in rural areas, and because Kintampo Health Research Centre (KHRC) offered an outstanding platform for scientific collaboration and study execution.

\section{METHODS}

\section{Study design}

Thirty-five communities were randomised into three study arms (LPG, improved biomass stove and control). These communities comprised all population centres in the Kintampo North and South districts of Ghana. Pilot studies established the feasibility of study procedures and demonstrated that intervention cookstoves were capable of reducing exposure in controlled settings. Randomisation at the level of the community was carried out using a coarsened exact matching procedure and was implemented by an independent statistician. ${ }^{10} 11$ The study implementation team learnt of community study arm allocation after all study personnel were recruited and assigned to their respective clusters. Given the clusterrandomised nature of the design and the highly visible nature of the intervention, potentially eligible women knew their intervention status at the time of recruitment.

The trial was implemented by a team from the KHRC and Columbia University. The trial was undertaken in accordance with the Declaration of Helsinki. The protocol was registered with ClinicalTrials.gov, and has been previously published. ${ }^{12}$ The trial was funded by the National Institutes of Health, the Global Alliance for Clean Cookstoves (now known as the Clean Cooking Alliance) and the Thrasher Research Fund. The funders played no role in study design, collection, analysis and interpretation of data, nor manuscript preparation. All of the authors vouch for adherence to trial protocol, completeness and veracity of data and analyses presented. All of the pregnant women provided written informed consent for their and their child's study participation. Anonymised participant data are available on request. No significant changes to methods were made after study commencement.

The selection of the intervention cookstoves was developed through extensive piloting and engagement with members of the communities where GRAPHS took place. Community members also provided feedback on exposure assessment procedures. Awareness about the health risks of HAP is not widespread in our study communities, so we did not involve study participants in the choice of outcome measures. Results of the study have been shared in community meetings, and the investigator team is engaged in ongoing research addressing household energy policy in Ghana.

\section{Participants}

The great majority of households in the study region farm for a living and cook with solid fuels. Wood was by far the dominant fuel at baseline, with 1334 out of 1414 participants reporting wood as their primary fuel. The balance reported charcoal as the primary fuel $(n=56)$ or had missing data $(n=9)$. About half of the study participants (669 out of 1414) reported using charcoal as a secondary fuel, and 26 households reported crop residue as secondary. The balance of households reported no secondary fuel use. Fuel use patterns did not differ significantly across study arms. We did not observe any LPG, kerosene or electric cooking at baseline. Low birth weight and respiratory infections are public health concerns in the region. ${ }^{13}$ Solid fuel use has remained stable over time in rural areas in Ghana, and we observed no changes in cooking practices in our control group or 
in non-intervention households in intervention communities. Fieldworkers carried out pregnancy surveillance in each study community and referred pregnant women to trained midwives for confirmation of pregnancy and establishment of gestational age by transabdominal ultrasound (SonoSite S180). Study midwives underwent intensive pre-trial ultrasound training and ongoing image review and quality control by a US-based obstetrician during the study as previously described.$^{14}$ Pregnant individuals were eligible to be enrolled if they were the primary cook in their household; were pregnant with a live, intrauterine fetus; were $\leq 24$ weeks gestation (to allow for delivery of intervention by 28 weeks at the latest); and were non-smokers. Women with a multiple gestation identified at the time of the screening ultrasound were excluded. Detailed eligibility criteria and screening procedures are available in the study protocol. ${ }^{12}$ Essentially all households in our study area relied primarily on solid fuels for cooking, so we did not screen for cooking fuel at enrolment. ${ }^{15}$

\section{Cookstove interventions}

Communities were assigned to one of two cookstove interventions or to the control arm (online supplemental figure 1). All enrolled participants received a mosquito bed net and health insurance. In the LPG intervention arm, participants received a two-burner LPG cookstove (Ghana Cylinder Manufacturing Company, Accra, Ghana), two $14.5 \mathrm{~kg}$ LPG cylinders, and monthly LPG deliveries for the duration of study enrolment at no cost to study participants. Additional LPG fuel was available as needed. In the improved biomass stove arm, participants received two single-burner BioLite HomeStove forced draft wood fuel cookstoves (BioLite, Brooklyn, New York, USA). The improved biomass stove reduces emissions via a thermoelectric-powered fan, which blows air into the combustion chamber to improve combustion efficiency, and stove geometry, which increases heat transfer efficiency. The BioLite has a side opening for fuel, and thus can accommodate wood fuels similar to those used in the traditional three-stone fires with minimal differences in processing. BioLite stoves are not suitable for burning charcoal or crop residue. Control participants continued to cook with the traditional biomass stoves and fuels. Fieldworkers encouraged intervention cookstove use, checked stove condition and conducted maintenance and repairs as necessary during weekly visits with each participant. Similar visits to the control participants were framed as bed net visits. Participants remained on study from enrolment through the end of the child's first year of life or until the end of surveillance in March 2016. On study completion, participants in the control and BioLite arms received a two-burner LPG cookstove and two 14.5 kg LPG cylinders.

\section{Efficacy endpoints}

The co-primary endpoints of the trial were birth weight among liveborn infants born at 28 weeks or later gestational age; and physician-assessed severe pneumonia in the first 12 months of life as defined in the WHO Integrated Management of Childhood Illnesses handbook (IMCI). ${ }^{16}$ Secondary trial birth endpoints included birth length and head circumference, preterm birth (defined as $\geq 28$ and $<37$ completed weeks gestation at delivery), low birth weight (defined as birth weight $<2500 \mathrm{~g}$ ) and small for gestational age (defined as $<10$ th percentile for gestational age) ${ }^{17}$ (see online supplemental material for detailed definitions). Secondary trial pneumonia endpoints included physician-diagnosed pneumonia and a composite outcome of fieldworker-diagnosed and physician-diagnosed pneumonia and severe pneumonia (again using IMCI definitions).

Community-based fieldworkers measured birth anthropometrics within 24 hours of birth at the place of delivery (home or facility). Birth weight was measured to the nearest $0.01 \mathrm{~kg}$ (Tanita digital scale model BD-590, Tanita, Illinois, USA) after standardising the scale to a $1 \mathrm{~kg}$ weight. Birth weight was considered missing if the fieldworkers were unable to measure birth weight within 72 hours of birth. Birth length was measured with use of the Ayrton Infantometer Model M-200 (Ayrton, Minnesota, USA) and recorded to the nearest $0.1 \mathrm{~cm}$ and head circumference measured to the nearest $0.1 \mathrm{~cm}$ using a paper tape (a 'lasso') from the Child Growth Foundation in London.

Pneumonia surveillance involved active (weekly fieldworker surveillance) and passive (self-referral) methods. Community-based fieldworkers trained in the IMCI guidelines made weekly home visits to identify potential pneumonia cases. The IMCI defines pneumonia as cough or difficulty breathing plus elevated respiratory rate ( $60 \mathrm{breaths} / \mathrm{min}$ in children aged $0-2$ months $(56$ days) or 50 breaths/min in children aged 2-12 months). Severe pneumonia was defined as pneumonia in a child less than 2 months of age or pneumonia plus the presence of oxygen saturation of less than $90 \%$ as measured by pulse oximetry, chest wall indrawing, stridor or any general danger sign (including convulsions, vomiting or inability to drink or breast feed, lethargy or unconsciousness). Any child with fieldworker-diagnosed pneumonia, or who was otherwise unwell, was brought to a central medical clinic for physician evaluation. Communitybased fieldworkers also facilitated self-referrals any time a parent felt that their child was ill. The study provided transportation and paid for incidental expenses related to all clinical visits. At the central clinic, study physicians trained in IMCI guidelines diagnosed pneumonia and severe pneumonia. The primary pneumonia outcome was physician-diagnosed severe pneumonia, without the use of chest radiograph or ultrasound.

Due to the visible nature of the intervention, fieldworkers were not blinded to study intervention arm Physicians examined study children at a central clinic and were unaware of study intervention assignment. Study physicians immediately began treatment for pneumonia or other diagnosed illness, including hospital 
admission where appropriate. As with prior studies, ${ }^{4}$ children were not considered to have a new pneumonia episode if a repeat diagnosis occurred within 21 days of a prior pneumonia episode. If the initial assessment diagnosed pneumonia and a repeat assessment within 21 days diagnosed severe pneumonia, the pneumonia episode was reclassified as severe. The secondary composite pneumonia outcome of fieldworker-diagnosed and physician-diagnosed pneumonia included physicianassessed pneumonia cases as described above in addition to fieldworker-assessed pneumonia cases where the child did not receive study physician assessment within 7 days of fieldworker assessment. In cases where a fieldworker diagnosis and subsequent physician diagnosis occurring within 7 days did not agree (eg, the fieldworkerdiagnosed pneumonia and the physician did not), the physician diagnosis was used.

\section{Air pollution exposure assessment}

We measured 72-hour personal exposures to carbon monoxide (CO) using Lascar EL-USB-CO sensors (Lascar Electronics, Erie, Pennsylvania, USA) and to particulate matter less than 2.5 microns in diameter $\left(\mathrm{PM}_{2.5}\right)$ using microPEM monitors (RTI, Research Triangle Park, North Carolina, USA). Antenatal CO monitoring occurred at enrolment prior to deployment of intervention cookstove, 3 weeks after intervention cookstove deployment, and at two additional time points evenly spaced over the remaining antenatal period. Postnatal CO monitoring of both the child and the mother took place 1, 3 and 9 months post partum. We measured $\mathrm{PM}_{2.5}$ in a subset of adult study participants during the second $\mathrm{CO}$ session (prenatally, 3 weeks post enrolment) and/or postnatally at child age 3 months. Budget limitations dictated the $\mathrm{PM}_{2.5}$ sample. Exposure assessment methods and results have been previously published. ${ }^{18}$

\section{Additional trial assessments}

Maternal age, parity and medical history were assessed at enrolment through questionnaires. Maternal height and weight were measured on enrolment and used to determine maternal body mass index (BMI). Enrolment questionnaires determining household assets were used to construct an asset index as a proxy for wealth (see online supplemental material). Weekly fieldworker visits from enrolment through delivery captured the number of antenatal visits. Infant sex and date of delivery were recorded at birth. Fieldworkers and physicians recorded dates of pneumonia assessments which were used to determine month of pneumonia and age of child at the time of pneumonia diagnosis. At each prenatal and postnatal fieldworker visit, continued use of intervention cookstove was assessed via questionnaire.

\section{Statistical analysis}

Power calculations for GRAPHS have been previously published. ${ }^{12}$ The estimated sample size was 1415 pregnant women, with randomisation yielding 365 participants in the LPG arm (across 9 clusters), 525 participants in the improved biomass arm (across 13 clusters) and 525 participants in the control arm (across 13 clusters). For the birth weight outcome, sample size calculations were based on estimated effect sizes (Cohen's D) of 0.32 for the improved biomass arm and 0.40 for the LPG arm, ${ }^{19} 20$ $15 \%$ attrition and a two-sided type 1 error rate of $5 \%$. We estimated that 1415 enrolled pregnancies would yield 1225 participants reaching age 1 . For pneumonia, we used an effect size derived from work in the Gambia, ${ }^{21}$ and, with the same attrition and type 1 error rate as above, computed power of 0.98 for the LPG arm, and 0.89 for the improved biomass arm.

Primary analyses were performed according to the intention-to-treat principle. First, we used linear regression models to assess differences in birth weight by study arm. Cluster-robust SE estimates (at the village level) were employed to account for the cluster-randomised nature of the intervention deployment. We additionally conducted secondary analyses that included adjustment for asset index; maternal BMI, age and parity; number of antenatal care visits dichotomised around the median (four visits); and infant sex. Additional continuous birth outcomes (head circumference, birth length and gestational age at delivery) were analysed in the same manner as birth weight. Categorical birth outcomes (preterm birth, low birth weight, small-for-gestational-age and neonatal death occurring within 7 days of birth) were analysed using modified Poisson regressions with robust SE using the 'sandwich' estimator.

Any infant born alive and with at least one fieldworker pneumonia surveillance visit was included in the pneumonia analyses. Primary pneumonia analyses were performed according to the intention-totreat principle. We employed generalised estimating equation (GEE) logistic regression models with exchangeable correlation structure and cluster-robust $\mathrm{SE}$ estimates to assess the difference in incidence of physician-assessed severe pneumonia in the first year of life per child-days. GEE was used to account for both multiple episodes within a child and the villagelevel intervention deployment. ${ }^{22}$ Secondary analyses adjusted for an asset index, month of delivery, month of pneumonia event, child sex and child age. These same models were used to examine the effect of cookstove intervention on physician-assessed pneumonia and composite outcomes of fieldworker-assessed and physician-assessed pneumonia and severe pneumonia. We additionally conducted survival analyses using Cox proportional-hazard models for the time to the first incident of physician-diagnosed severe pneumonia, pneumonia or composite pneumonia outcomes, considered separately, to examine group differences in the risk, with and without adjustment of child sex, month of delivery and an asset index. HRs for group comparisons were derived from estimated model parameters, and their CIs were based on robust sandwich estimate of variance of parameter estimates to 
account for possible within-cluster (village) correlation among the children. Analyses were carried out in R V.3.6.0 and SAS V.9.4.

The funder of the study had no role in study design, data collection, data analysis, data interpretation or writing of the report. All authors had full access to all the data in the study and had final responsibility for the decision to submit for publication.

\section{RESULTS}

Between 14 April 2014 and 20 August 2015, we screened 1714 women and excluded 300 who were either not pregnant $(\mathrm{N}=53)$, carrying twin gestations $(\mathrm{N}=22)$ or who had pregnancies at gestational age of over 24 weeks $(\mathrm{N}=225)$. Of the 1414 households with an eligible pregnant woman that underwent randomisation, 361 were assigned the LPG intervention, 527 were assigned the

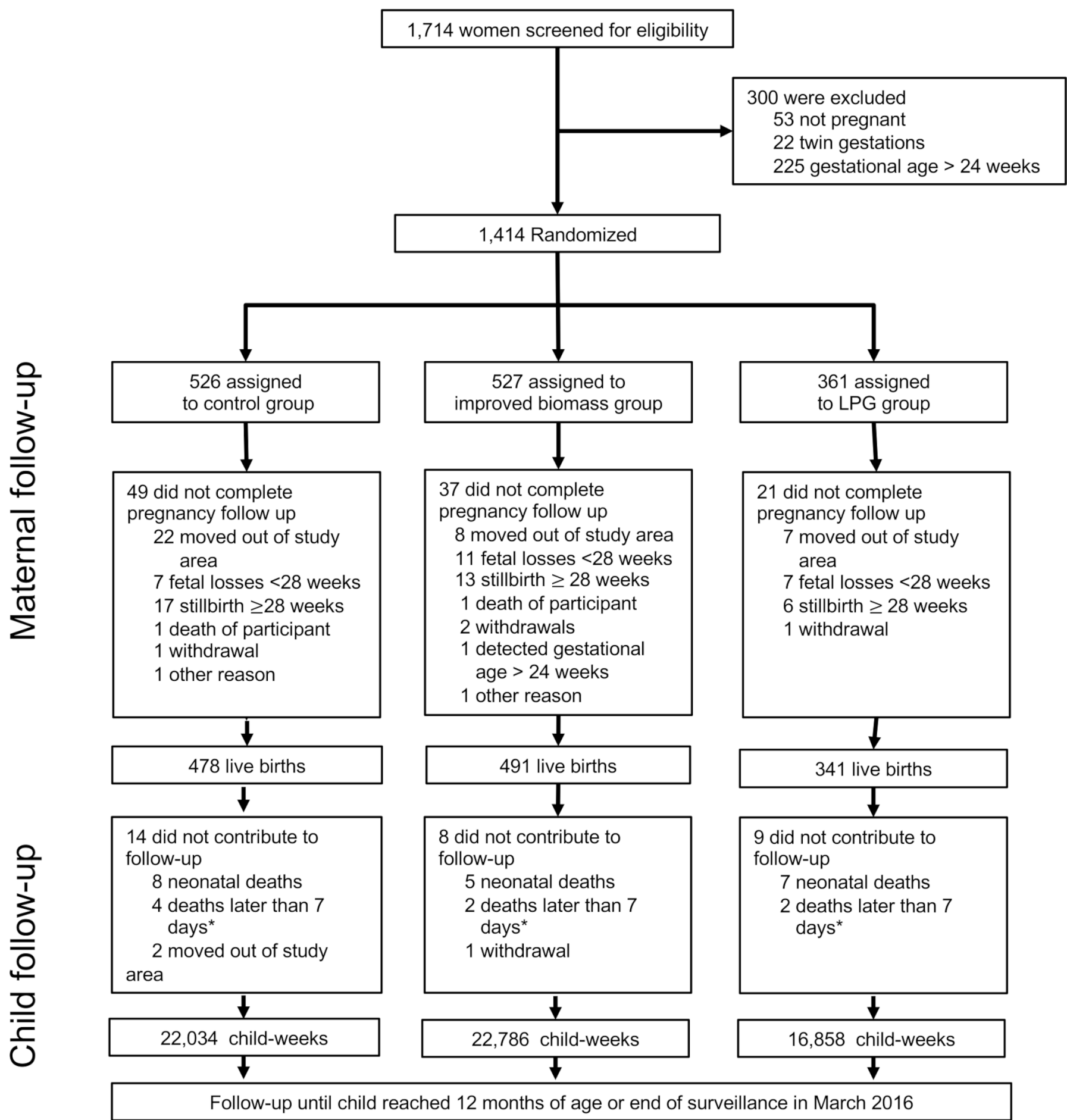

Figure 1 Trial profile. *Deaths of children at age $>7$ days who did not have any recorded fieldworker follow-up. LPG, liquefied petroleum gas. 
Table 1 Characteristics of the participants at baseline*

\begin{tabular}{|c|c|c|c|}
\hline Characteristic & Control $(\mathrm{N}=526)$ & Improved biomass ( $\mathrm{N}=527)$ & LPG (N=361) \\
\hline \multicolumn{4}{|l|}{ Maternal characteristics } \\
\hline Age-years & $27.3 \pm 7.4$ & $28 \pm 7.2$ & $26.9 \pm 6.7$ \\
\hline $\begin{array}{l}\text { Gestational age at enrolment- } \\
\text { weeks } \dagger\end{array}$ & $16.2 \pm 4.3$ & $16.1 \pm 4.4$ & $14.6 \pm 4.2$ \\
\hline Range & $6-26$ & $6-24$ & $6-23$ \\
\hline Body mass index $\ddagger$ & $23.2 \pm 3.1$ & $23.5 \pm 3.2$ & $23 \pm 3.3$ \\
\hline Parity & $2.6 \pm 2.1$ & $2.8 \pm 2.3$ & $2.5 \pm 2.1$ \\
\hline \multicolumn{4}{|l|}{$\begin{array}{l}\text { Health history-no. / total no. } \\
(\%) \S\end{array}$} \\
\hline Anaemia & $13 / 526(2.5)$ & $12 / 527(2.3)$ & 6/361 (1.7) \\
\hline Hypertension & $11 / 526(2.1)$ & $7 / 527(1.3)$ & $5 / 361(1.4)$ \\
\hline Diabetes & 0/526 (0) & $1 / 527(0.2)$ & $1 / 361(0.3)$ \\
\hline HIV & $1 / 526(0.2)$ & $0 / 527(0)$ & $1 / 361(0.3)$ \\
\hline $\begin{array}{l}\text { Completed primary education - } \\
\text { no. / total no. (\%) }\end{array}$ & $334 / 525(63.6)$ & $325 / 527(61.7)$ & $191 / 360(53.1)$ \\
\hline Is married-no. / total no. (\%) & $277 / 525$ (52.8) & $309 / 527$ (58.6) & $191 / 360(53.1)$ \\
\hline \multicolumn{4}{|l|}{$\begin{array}{l}\text { Pre-intervention personal CO } \\
\text { exposure-ppm }\end{array}$} \\
\hline Mean $\pm S D$ & $1.49 \pm 1.20$ & $1.46 \pm 1.20$ & $1.56 \pm 1.15$ \\
\hline Median (IQR) & $1.17(0.62-2.09)$ & $1.17(0.63-1.94)$ & $1.29(0.70-2.13)$ \\
\hline \multicolumn{4}{|l|}{ Household characteristics } \\
\hline Asset index & $0.2 \pm 2.2$ & $-0.0 \pm 1.8$ & $-0.2 \pm 1.8$ \\
\hline
\end{tabular}

*Plus-minus values are means \pm SD. Across arms, differences are noted in maternal age, gestational age at enrolment, parity, maternal education, marital status and asset index.

†Gestational age at enrolment established by ultrasound.

†The body mass index is weight in kilograms divided by the square of the height in metres.

$\S$ Health history is based on self-reported responses to questions framed as 'Has a doctor ever diagnosed you with (health condition)?' ๆPersonal CO exposure derived from valid deployments truncated at 48-hours for analysis reported in parts per million (ppm). Due to QA/ QC, 146, 165 and 101 participants in the control, improved biomass and LPG arms did not have pre-intervention personal CO exposure assessments.

CO, carbon monoxide; LPG, liquefied petroleum gas.

improved biomass cookstove intervention and 526 were assigned control (figure 1). Fewer clusters were assigned intentionally to the LPG arm because we assumed that it would result in a greater reduction in pollution exposure, and would therefore require a smaller sample to detect an effect. The baseline characteristics are shown in table 1 . The study groups demonstrated balance in key characteristics such as maternal BMI, health history and baseline exposures to CO (a key component of HAP). Across arms, we note differences in maternal age, gestational age at enrolment, parity, maternal education and marital status. Our overarching conclusion from table 1 is that our randomisation achieved reasonable balance, but that adjusted analyses are warranted to account for small differences in baseline characteristics.

Across the study period, participants reported using their intervention stoves to cook their main meal the previous day during approximately $87 \%$ of weekly visits to households in the LPG study arm ( 25000 visits) and $69 \%$ of visits to households in the improved biomass study arm ( $\sim 34000$ visits) (online supplemental figure
2). We did not observe control households switching to cleaner cookstoves or fuels during the study period.

The study cohort resulted in 340 (94.2\% of enrolled women) live births 28 weeks or later gestational age in the LPG arm, 491 (93.2\%) in the improved biomass cookstove arm and 475 (90.3\%) in the control arm, including four sets of twins identified at delivery (one in LPG, one in improved biomass and two in control). As these twin pregnancies were identified only after delivery, the mother-twin triads were followed through the first year of life and data included in all analyses. Average gestational age at delivery was: $39 \pm 1.7$ weeks in the LPG arm, $39.3 \pm 1.5$ weeks in the improved biomass arm and $39.2 \pm 1.8$ in the control arm. There were 245 (51\%), 233 (45\%) and 174 $(51 \%)$ female newborns in the LPG, improved biomass and control arms, respectively.

Birth weight was measured for 331 (97.4\% of live births), $474(96.5 \%)$ and $462(97.2 \%)$ newborns in the LPG, improved biomass and control arms, respectively. The mean birth weights by study arm were: $2867 \pm 470 \mathrm{~g}$ for LPG, $2904 \pm 458 \mathrm{~g}$ for improved biomass cookstove 
arm and $2894 \pm 454$ for controls. There was no difference in birth weight between study arms. The unadjusted $(\mathrm{N}=1267)$ and adjusted $(\mathrm{N}=1258)$ difference in mean birth weight for LPG newborns was $-27 \mathrm{~g}$ (95\% CI -111 to $58, \mathrm{p}=0.54)$ and $-15 \mathrm{~g}$ ( $95 \% \mathrm{CI}-96$ to $66, \mathrm{p}=0.71)$ as compared with control newborns, respectively. The unadjusted and adjusted difference in birth weight for the improved biomass cookstove arm newborns was $10 \mathrm{~g}$ (95\% CI -63 to $84, \mathrm{p}=0.78)$ and $-8 \mathrm{~g}(95 \% \mathrm{CI}-81$ to 64 , $\mathrm{p}=0.82$ ) as compared with control newborns, respectively (table 2).

Analysis of secondary birth outcomes did not demonstrate differences in head circumference or birth length between study arms. There were 8 (2\%), $11(2 \%)$ and 10 (1\%) fetal losses (loss at $<28$ weeks gestational age) and $6(2 \%), 13(2 \%)$ and $17(3 \%)$ stillbirths (lost or not born alive $\geq 28$ weeks gestational age) in the LPG, improved biomass and control arms, respectively. Unadjusted $(\mathrm{N}=1303)$ analyses suggested there were fewer preterm births in the improved biomass cookstove arm compared with control (12 (2\%) vs $24(5 \%)$ preterm births, or a risk ratio of 0.48 (95\% CI 0.25 to $0.93, \mathrm{p}=0.03)$ ); the adjusted $(\mathrm{N}=1294)$ difference was $0.50(95 \%$ CI 0.26 to 0.95 , $\mathrm{p}=0.03)$. No difference in preterm birth was detected between LPG and control arms. There was no difference in low birth weight, small for gestational age or neonatal deaths between study arms.

Of the 1306 live births at 28 weeks gestation or after, $40(3.4 \%)$ children died in the first year of life, or 33.6 deaths per 1000 live births. Of these, 26 occurred in the first 28 days of life (22.9 neonatal vs 10.7 post-neonatal deaths per 1000 live births).

For all 1306 live-born children, weekly fieldworker visits over the first year of life provided an opportunity for 67912 child-weeks of fieldworker follow-up. Pneumonia follow-up lasted through 22 March 2016, when the youngest GRAPHS child aged out of the study. In total, 61 $678(93.3 \%$, accounting for 1811 child-weeks lost due to under-1 mortalities) child-weeks of fieldworker follow-up (16 858, 22786 and 22034 in LPG, improved biomass and control arms, respectively) resulted in identification of 408 physician-diagnosed pneumonia cases $(115,155$ and 138 cases in LPG, improved biomass and control arms, respectively) and 127 physician-diagnosed severe pneumonia cases $(32,52$ and 43 in the LPG, improved biomass and control arms, respectively). There was no significant difference in physician-assessed severe pneumonia between study arms. The unadjusted and adjusted rate ratios for severe pneumonia in children in the LPG arm was 0.98 (95\% CI 0.58 to $1.70 ; \mathrm{p}=0.95$ ) and 0.98 (95\% CI 0.58 to $1.65 ; \mathrm{p}=0.93$ ) as compared with children in the control arm, respectively. The unadjusted and adjusted rate ratios for severe pneumonia in children in the improved biomass arm was 1.21 (95\% CI 0.78 to 1.90 ; $\mathrm{p}=0.39)$ and 1.19 (95\% CI 0.74 to $1.91 ; \mathrm{p}=0.48)$ in the LPG arm, as compared with children in the control arm, respectively (table 3 ).
Analysis of secondary pneumonia outcomes did not demonstrate differences in physician-assessed pneumonia or composite fieldworker-assessed and physicianassessed pneumonia or severe pneumonia between study arms. Unadjusted and adjusted survival analyses for incidence of child pneumonia using Cox proportionalhazard regressions did not demonstrate differences in physician-assessed or physician-assessed and fieldworkerassessed pneumonia and severe pneumonia between groups (figure 2, (online supplemental table 2).

Personal CO exposure assessments were performed for 515 women in the control arm, 520 in the improved biomass stove arm and 354 in the LPG arm. Prior to intervention, median personal $\mathrm{CO}$ exposures were 1.17 parts per million (ppm; IQR, 0.62-2.09), $1.17 \mathrm{ppm}$ (IQR, $0.64-1.94)$ and $1.30 \mathrm{ppm}$ (IQR, 0.70-2.13), respectively, for women in the control, improved biomass stove and LPG arms (table 1). Following intervention, personal $\mathrm{CO}$ exposure was lower in the LPG arm (median CO, $0.52 \mathrm{ppm}$; IQR, 0.20-1.16) and in the improved biomass stove arm (median CO, $0.74 \mathrm{ppm}$; IQR, 0.33-1.47) as compared with in the control arm (median CO, 0.82 ppm; IQR, 0.37-1.65).

Valid post-intervention personal prenatal or postnatal $\mathrm{PM}_{2.5}$ exposure assessments were performed in 202, 321 and 356 women in LPG, improved biomass and control arms, respectively. Personal $\mathrm{PM}_{2.5}$ exposures in the LPG arm (median $\mathrm{PM}_{2.5}, 45 \mu \mathrm{g} / \mathrm{m}^{3}$; IQR, 32-65) were lower than those in the control arm (median $\mathrm{PM}_{2.5}, 67 \mu \mathrm{g} /$ $\mathrm{m}^{3}$; IQR, 46-97). There was no difference in personal $\mathrm{PM}_{2.5}$ exposures between improved biomass cookstove (median $\mathrm{PM}_{2.5}, 67 \mu \mathrm{g} / \mathrm{m}^{3}$; IQR, 44-94) and control arms (figure 3).

No harms or adverse events were observed as a result of trial procedures.

\section{DISCUSSION}

We carried out a cluster-randomised controlled trial assessing the benefits of clean cooking interventions initiated during pregnancy and continued for the first year of the infant's life. In intention-to-treat analyses, we found no evidence that either improved biomass or LPG cookstoves led to improvements in birth weight or to reductions in severe pneumonia risk. These null findings extended to secondary obstetrical and pneumonia outcomes, including anthropometrics at birth, preterm birth, low birth weight, small for gestational age and alternative pneumonia case definitions (with the exception of a reduction in the risk of preterm birth in the improved biomass arm, which we attribute to chance).

Worldwide, ALRI is a leading cause of death in children under 5 years of age and HAP pollution exposure is the second leading risk factor. Despite the epidemiological evidence supporting an exposure-response association, evidence for the efficacy of a cooking intervention to reduce ALRI risk is mixed. Two trials-RESPIRE in Guatemala $^{4}$ and a large study in Rwanda ${ }^{8}$-found some 


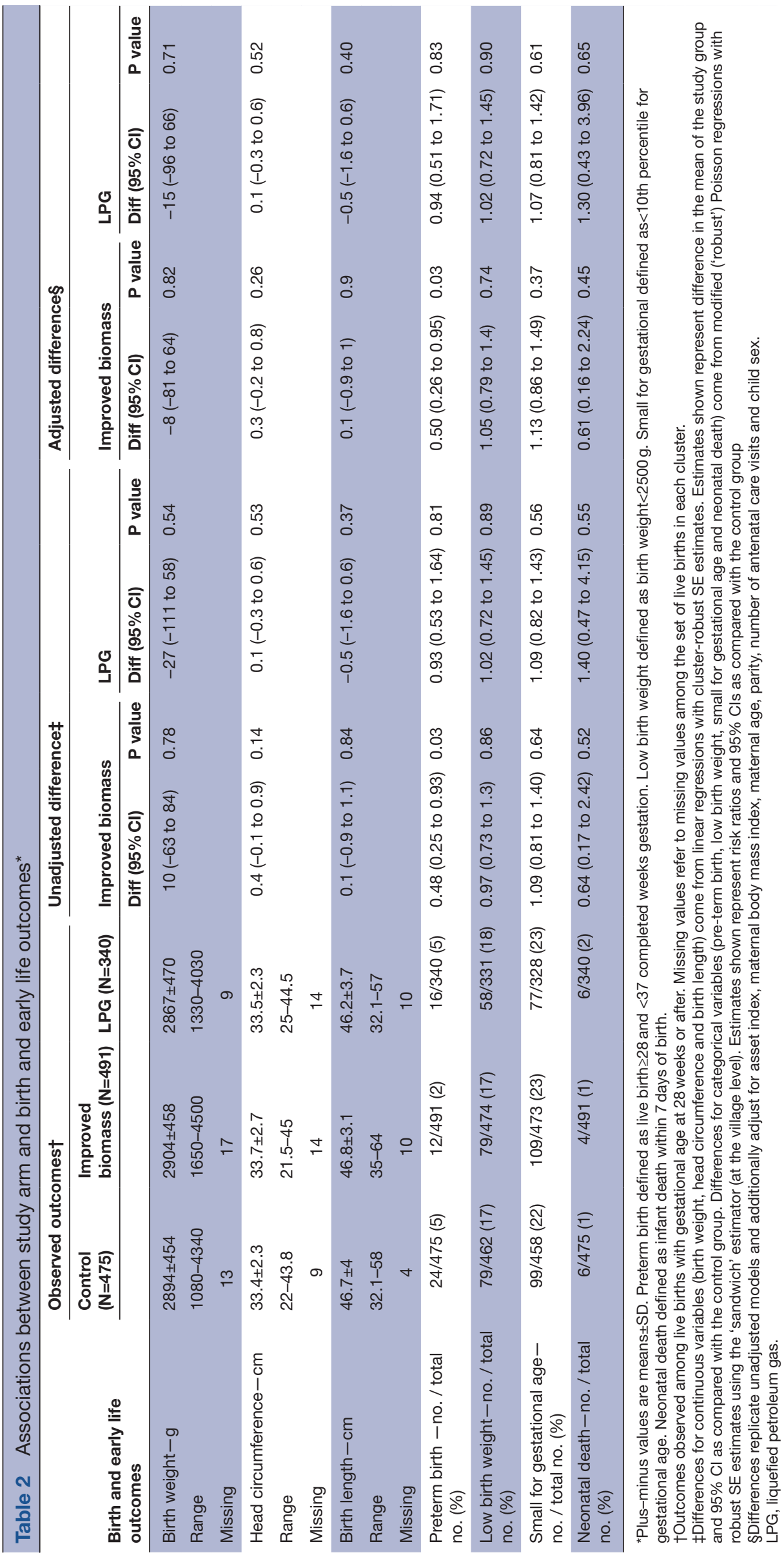


evidence of reduced ALRI risk from improved biomass interventions. RESPIRE deployed a chimney stove intervention that resulted in a 50\% reduction from 2.2 to 1.1 ppm CO exposure. Intention-to-treat analyses found a $22 \%$ (rate ratio (RR) $0.78,95 \%$ CI 0.59 to $1.06, p=0.10$ ) and a $33 \%$ (RR $0.67,95 \%$ CI 0.45 to $0.98, p=0.04$ ) reduction in physician-diagnosed pneumonia and severe pneumonia, respectively. Kirby et alstudied a natural draft rocket-style stove versus no intervention in Rwanda and observed a $25 \%$ reduction in reported child ALRI (RR 0.75, 95\% CI 0.60 to $0.93, \mathrm{p}=0.01$ ) without a measured reduction in cook or child-level $\mathrm{PM}_{2.5}$ exposure. Conversely, the CAPS trial in Malawi ${ }^{5}$ and a smaller trial in Peru, ${ }^{6}$ both evaluating improved biomass stove interventions, found no benefit on child ALRI. Direct comparison with these prior studies is hampered by differences in baseline air pollution exposures, inclusion criteria and intervention cookstoves.

Similar to ALRI, data has been mixed from interventional studies about whether improved cook stoves can impact birth outcomes. Investigators from RESPIRE reported that birth weight was $89 \mathrm{~g}$ heavier among those using a chimney stove in the trial; however, the CI was wide and crossed 0 (95\% CI $-27 \mathrm{~g}$ to $204 \mathrm{~g}){ }^{23}$ Furthermore, as recruitment into RESPIRE included households with either a pregnant woman or infant $<4$ months, only 266 of the 534 households recruited included a pregnant woman and the benefits of randomisation in balancing potential drivers of birth weight differences might have been lost. Researchers in rural Nepal were unable to demonstrate a change in birth weight or other pregnancy outcomes in two sequentially conducted randomised trials evaluating first an improved and vented biomass stove and second LPG stoves. ${ }^{7}$ In contrast, benefits were reported from a trial conducted in Ibadan, Nigeria, that individually randomised 324 pregnant women to use of an ethanol stove versus continued use of traditional fuels (kerosene and firewood). ${ }^{24}$ The $88 \mathrm{~g}$ improvement in birth weight was not significantly different from the control group (95\% CI -18 to 194) in unadjusted intention-to-treat analyses. The authors report a significant improvement in birth weight from adjusted models (197 grams, 95\% CI 25 to 368) accounting for some baseline differences that were imbalanced despite randomisation. Notably, the ethanol stove intervention did not significantly impact personal exposure to $\mathrm{PM}_{2.5}$ as compared with control. The GRAPHS LPG intervention, by contrast, reduced exposure to $\mathrm{CO}$ and $\mathrm{PM}_{2.5}$, yet did not perform as hypothesised with regards to improvements in birth weight or other pregnancy outcomes. While we did observe a reduction in preterm birth in mothers using the biomass stove, we are unable to explain this isolated benefit and cannot exclude the possibility that the significant result was due to chance, particularly given the low preterm birth rate observed in our population.

The null trial result did not appear to be driven by poor uptake of the intervention. Households randomised to the LPG arm received unlimited LPG fuel for the study 

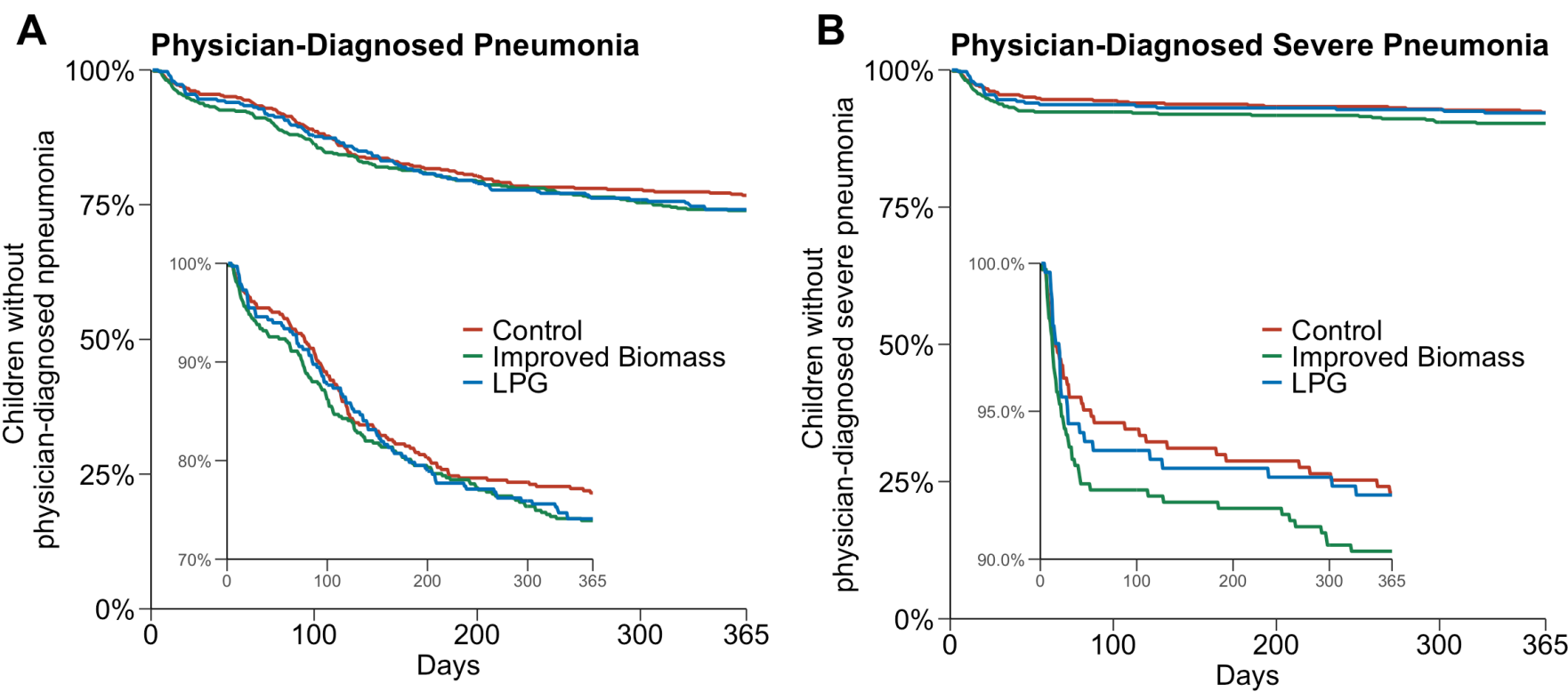

\section{No. at Risk}

$\begin{array}{rlll}\text { Control 464 } & 402 & 355 & 284 \\ \text { I. Biomass 483 } & 407 & 341 & 296 \\ \text { LPG 332 } & 289 & 257 & 241\end{array}$
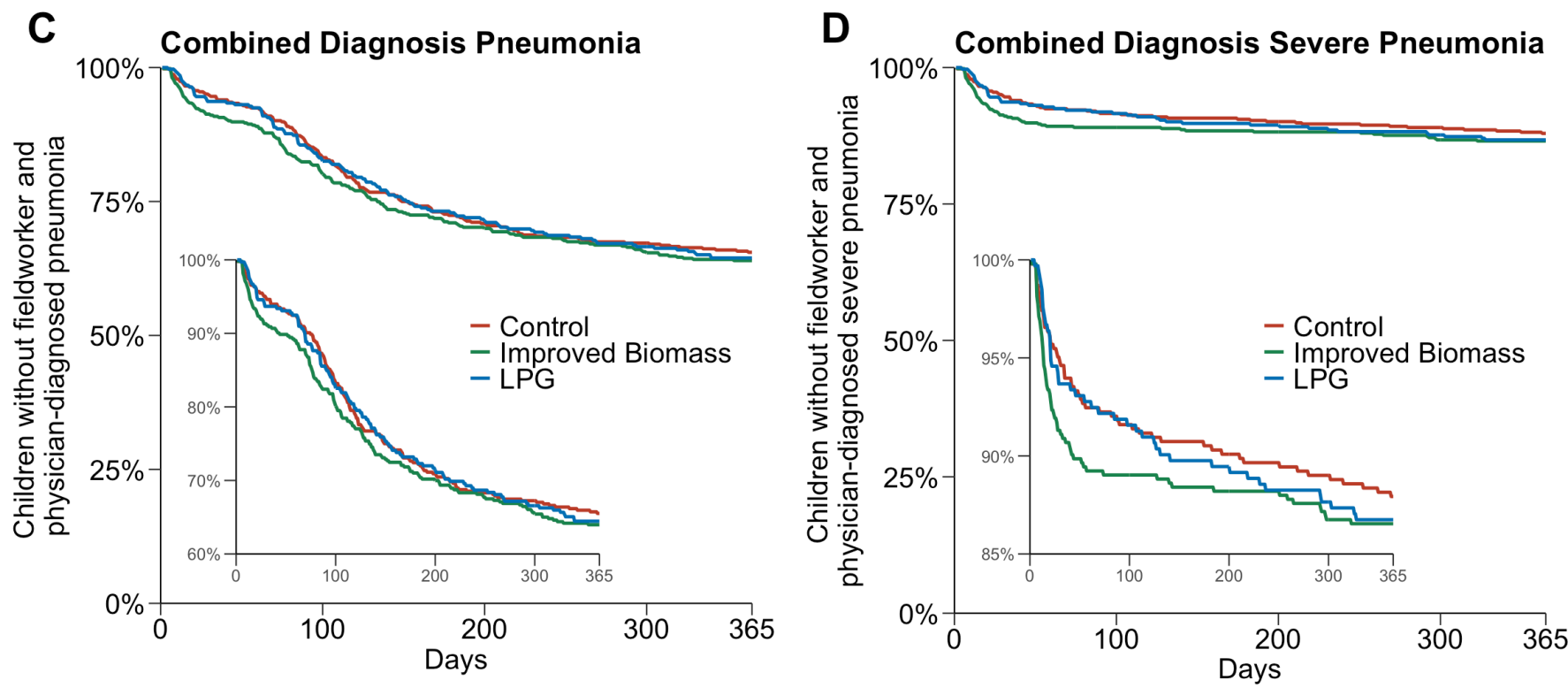

No. at Risk

$\begin{array}{rlll}\text { Control 464 } & 378 & 312 & 246 \\ \text { I. Biomass 483 } & 378 & 299 & 254 \\ \text { LPG 332 } & 273 & 234 & 212\end{array}$

\section{No. at Risk}

$\begin{array}{llll}464 & 417 & 401 & 327 \\ 483 & 421 & 379 & 342 \\ 332 & 303 & 293 & 277\end{array}$

Figure 2 Time to first event of: (A) Physician-diagnosed pneumonia; (B) physician-diagnosed severe pneumonia; (C) combined physician-diagnosed and fieldworker-diagnosed pneumonia; and (D) combined physician-diagnosed and fieldworker-diagnosed pneumonia by control, improved biomass or liquefied petroleum gas (LPG) cookstove study arm. Cox proportional-hazard models adjusting for asset index, month of delivery, child sex and child age demonstrated no difference in groups regardless of pneumonia outcome considered.

duration. For both intervention arms, weekly fieldworker check-ins facilitated stove repairs and encouraged use. Fieldworkers assessed use of the intervention stoves every week and found that on average $87 \%$ and $69 \%$ of households in the LPG arm and improved biomass stove arm, respectively, reported using the intervention stove. These results may be subject to recall bias and objective data, such as from stove use monitoring devices, was not obtained. However, these data do suggest that on average the uptake of stove intervention was robust. 


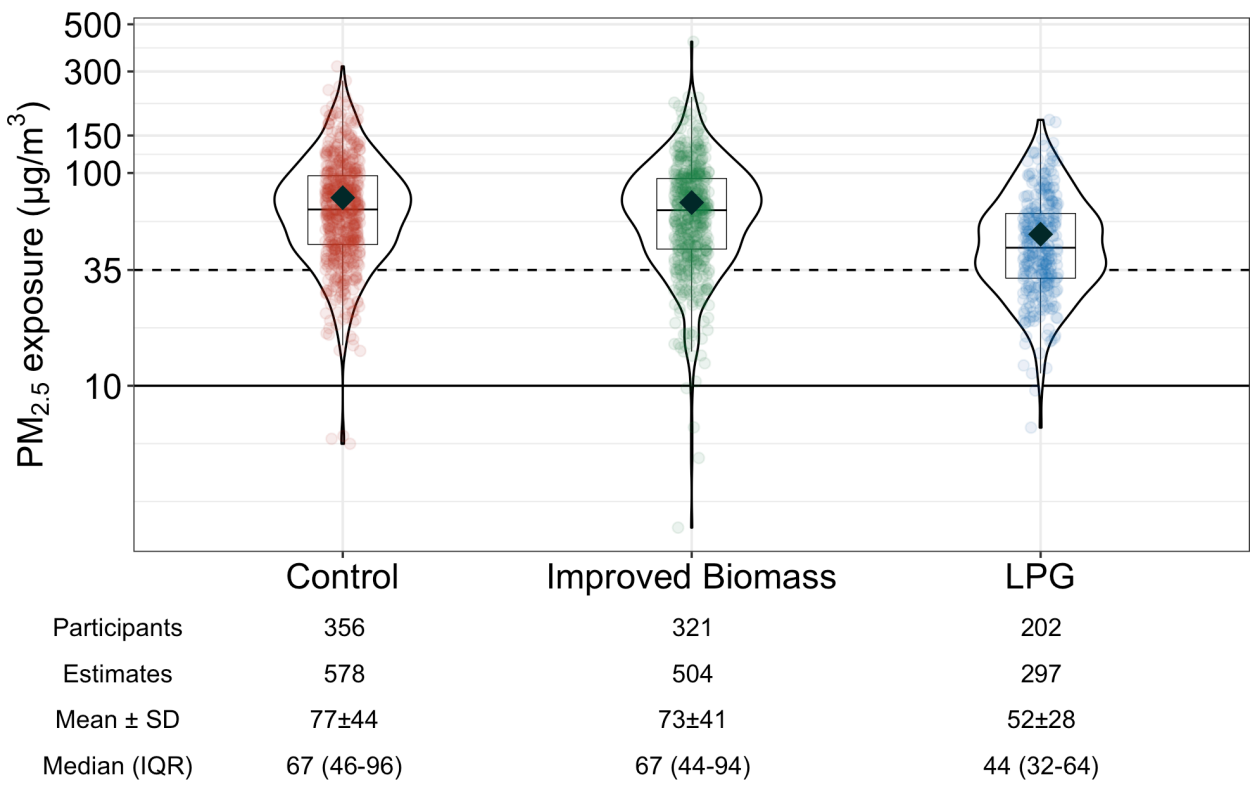

Figure 3 The distribution of maternal personal fine particulate matter $\left(\mathrm{PM}_{2.5}\right)$ exposure during the GRAPHS post-intervention period. Violin plots show the density of air pollution exposures, boxplots show the median and IQR, the mean exposure is shown with black diamonds for each study arm and partially transparent dots show all 48-hour estimates. The dotted line represents the WHO interim-1 guideline for annual $\mathrm{PM}_{2.5}$ exposure $\left(35 \mu \mathrm{g} / \mathrm{m}^{3}\right)$ and solid line is the WHO guideline for annual $\mathrm{PM}_{2.5}$ exposure $\left(10 \mu \mathrm{g} / \mathrm{m}^{3}\right)$. In the control arm, 174 women had one $\mathrm{PM}_{2.5}$ exposure estimate, 145 had two exposure estimates

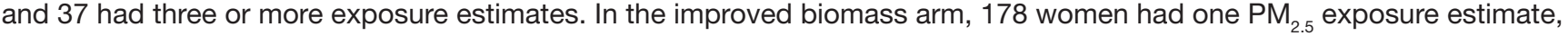
106 had two exposure estimates and 37 had three or more exposure estimates. In the liquefied petroleum gas (LPG) arm, 125 women had one $\mathrm{PM}_{2.5}$ exposure estimate, 59 had two exposure estimates and 18 had three or more exposure estimates. All estimates, including multiple observations per participant, are plotted and contribute equally to summary statistics. Summary statistics 'mean \pm SD' and 'median (IQR)' are 48-hour $\mathrm{PM}_{2.5}$ concentration estimates with units $\mu \mathrm{g} / \mathrm{m}^{3}$. GRAPHS, Ghana Randomized Air Pollution and Health Study; $\mathrm{PM}_{2.5}$, particulate matter less than 2.5 microns in diameter.

The central question, therefore, raised by the study results is why the intervention failed to improve health. In a laboratory setting, the GRAPHS LPG intervention stove reduces $\mathrm{PM}_{2.5}$ and $\mathrm{CO}$ emissions to levels meeting WHO emission rate targets, ${ }^{25}$ yet our intervention did not improve birth weight or reduce pneumonia risk. We hypothesise that our cookstove interventions, as deployed in the field, failed to reduce exposures enough to improve health outcomes. The WHO Interim-1 Target for annual average $\mathrm{PM}_{2.5}$ exposure is $35 \mu \mathrm{g} / \mathrm{m}^{3}{ }^{26}$ but intervention households in our study exceeded this target despite reported consistent use.

Several factors may explain this residual exposure in the LPG arm, and potentially also in the improved biomass arm-though for the improved biomass arm we cannot rule out the possibility that the stove itself was an important source of emissions. First, our householdlevel intervention left study participants exposed to emissions from neighbours' cooking, as has been suggested in previous field assessments of clean fuel stoves. ${ }^{27}$ Our study communities had high housing density (control communities mean (SD) persons within $50 \mathrm{~m}: 48.4$ (29.6); BioLite communities mean (SD): 48.6 (31.9); LPG communities mean (SD): 53.9 (35)) and, given the inclusion criteria requiring a pregnant participant, few households per community were enrolled. Second, LPG households may have continued to use their traditional biomass cookstoves. As described above, our weekly survey data suggests that this was rare in the LPG arm (13\% of meals), but we lack objective sensor-based measurements to rule out the possibility of persistent stove stacking. Third, emissions from other community or regional combustion sources, such as trash burning or agricultural burning, may have kept exposures high, but again our exposure assessment approach did not allow us to differentiate by source.

We also note that HAP exposure levels-both preintervention and post-intervention-are relatively low in our cohort, compared with the other trials discussed above. When we designed the GRAPHS study we viewed this exposure environment as a strength. We hypothesised that these levels implied that exposure reductions would occur on the steep part of the exposure response curve for pneumonia, which was estimated by Burnett et al to drop sharply below at low levels of $\mathrm{PM}^{2.5}$ exposure, but to be relatively flat at high levels. ${ }^{28}$ We cannot rule out the possibility that the ALRI shape of the exposure response curve does not follow this pattern, or that factors specific to central Ghana generate a different shape.

Our study did not enrol participants until the second trimester of pregnancy. Interventions to improve newborn and infant health may have greater effect if initiated earlier in pregnancy. If so, this would have significant implications for the design of clean household energy policies in LMIC settings. 
Observed infant, neonatal and post-neonatal mortality rates were only about half what has been reported for the broader Ghanaian population, suggesting that the totality of our intervention, with frequent interactions between participants and our study team and improved access to healthcare, may have improved infant health across study arms. If this was the case, it is possible that in the context of improved overall health the added benefit of a cleaner burning stove was not detectable. The 2011 Ghana Multiple Cluster Indicator Survey estimated infant mortality at 53 deaths per 1000 live births; neonatal (first month of life) and post-neonatal (age 1 month-first birthday) mortality rates were estimated at 32 and 21 deaths per 1000 live births. ${ }^{29}$ In the Brong Ahafo region where Kintampo is located, these numbers are even higher (66, 44 and 21 infant, neonatal and postneonatal, respectively, deaths per 1000 live births). In our cohort, weekly fieldworker follow-up allowed tracking of mortality over infancy. We observed lower infant mortality, evident in both the neonatal and post-neonatal periods (33.6, 22.9 and 10.7 infant, neonatal and postneonatal deaths per 1000 live births). The provision of insecticide-treated bed nets and health insurance to all mother-infant dyads and easy access to healthcare over the study period, including physician assessment and treatment, appears to have had a substantial effect on maternal and infant health. This overall effect may have reduced our ability to detect a meaningful impact of HAP interventions on health.

Our study has several strengths. GRAPHS was among the first trials to assess LPG as an alternative to improved biomass cookstoves, and to assess LPG alongside lowercost improved biomass interventions. As with RESPIRE, our study relied on active case finding and physicianassessed diagnosis for ALRI; other studies relied on community-assessed ALRI. We employed an active pneumonia surveillance programme wherein study children were assessed every week. This approach likely identified pneumonia cases early in their clinical course and potentially reduced the number of severe pneumonia cases we would have seen had this network not been in place. We acknowledge that active case finding could therefore be construed as a weakness. Active case finding was deemed ethical by our team as it is well-described that infectious mortality is related to time to treatment. Indeed, the infant mortality rate in our cohort was nearly half of country and regional trends, suggesting that this approach saved lives. Other important strengths of the study include ultrasound-assessed gestational age, digital measurement of birth weight even for in-home deliveries, extensive personal exposure monitoring and physician assessment of suspected pneumonia cases.

We also acknowledge limitations. Given the fieldworkers visited the households weekly and that our intervention was clustered by community, we were unable to blind fieldworkers to study arm. However, physician assessments were performed at a central clinic and physicians were unaware of study assignment. Participants reported stove use weekly, but these data may have inherent bias. Budget limitations prevented us from sensor-based stove use assessments, which would have addressed this potential bias. Our ALRI diagnosis would have been strengthened by objective diagnostic tests such as lung ultrasound or chest X-rays, however these were not readily available at our study site.

In conclusion, we found no evidence that a strategy of providing even the cleanest fuels to individual pregnant women in the context of antenatal care improved birth weight or reduced infant severe pneumonia. Air pollution levels in both intervention arms remained high, suggesting that meaningful health improvements from HAP interventions may require deployment of clean energy across entire communities.

\section{Author affiliations \\ ${ }^{1}$ Department of Environmental Health Sciences, Columbia University, New York, New York, USA \\ ${ }^{2}$ Kintampo Health Research Centre, Research and Development Division, Ghana Health Service, Kintampo North Municipality, Ghana \\ ${ }^{3}$ Division of Pulmonary, Critical Care and Sleep Medicine, Icahn School of Medicine at Mount Sinai, New York, New York, USA \\ ${ }^{4}$ Lamont-Doherty Earth Observatory, Columbia University, Palisades, New York, USA ${ }^{5}$ Fogarty International Center, National Institutes of Health, Bethesda, Maryland, USA ${ }^{6}$ Department of Environmental Medicine and Public Health, Icahn School of Medicine at Mount Sinai, New York, New York, USA \\ ${ }^{7}$ Institute for Health Metrics and Evaluation, University of Washington, Seattle, Washington, USA \\ ${ }^{8}$ Department of Biostatistics, Columbia University, New York, New York, USA ${ }^{9}$ Center for Maternal-Fetal Medicine, Department of Obstetrics and Gynecology, Beth Israel Deaconess Medical Center, Boston, Massachusetts, USA \\ ${ }^{10}$ University of Health and Allied Sciences, Ho, Ghana \\ ${ }^{11}$ Department of Environmental Health, Boston University, Boston, Massachusetts, USA}

\section{Twitter Darby W Jack @darbyjack}

Contributors DJ, PK, RW, BW and KPA initiated the study design. KPA, KA-N, EB-K, $\mathrm{MM}, \mathrm{SK}, \mathrm{FBO}, \mathrm{OA}, \mathrm{JAA}-\mathrm{a}, \mathrm{YAB}$ and SAE led trial implementation in Ghana. SC, AKQ, $\mathrm{DC}$ and $\mathrm{MM}$ led the exposure assessment. $\mathrm{SG}, \mathrm{XL}, \mathrm{KB}$ and $\mathrm{CFG}$ analysed the data with input from DJ and AGL. All authors contributed to refinement of the study protocol and approved the final manuscript.

Funding This study was funded by NIH R01 ES019547, NIH P30 ES009089, NIH S10 0D016219-01, Clean Cooking Alliance and Thrasher Research Fund. BW was supported by NIH K23 ES021471. AGL was supported by NIH K23 HL135349. CFG was supported by NIH T32 ES023770 and F31 ES031833. The findings and conclusions in this report are those of the authors and do not necessarily represent the official position of the US National Institutes of Health or Department of Health and Human Services.

Competing interests None declared.

Patient consent for publication Not required.

Ethics approval The study was approved by the Institutional Ethics Committee at Kintampo Health Research Centre and Ghana Health Service Ethics Review Committee, and Institutional Review Boards at Columbia University and Massachusetts General Hospital.

Provenance and peer review Not commissioned; externally peer reviewed.

Data availability statement Data are available upon request. Anonymised participant data that underlie the results reported in this analysis are available on request. Proposals should be directed to kwakupoku.asante@kintampo-hrc.org and to darby.jack@columbia.edu; to gain access, data requestors will need to sign a data access agreement.

Supplemental material This content has been supplied by the author(s). It has not been vetted by BMJ Publishing Group Limited (BMJ) and may not have been peer-reviewed. Any opinions or recommendations discussed are solely those 
of the author(s) and are not endorsed by BMJ. BMJ disclaims all liability and responsibility arising from any reliance placed on the content. Where the content includes any translated material, BMJ does not warrant the accuracy and reliability of the translations (including but not limited to local regulations, clinical guidelines, terminology, drug names and drug dosages), and is not responsible for any error and/or omissions arising from translation and adaptation or otherwise.

Open access This is an open access article distributed in accordance with the Creative Commons Attribution Non Commercial (CC BY-NC 4.0) license, which permits others to distribute, remix, adapt, build upon this work non-commercially, and license their derivative works on different terms, provided the original work is properly cited, appropriate credit is given, any changes made indicated, and the use is non-commercial. See: http://creativecommons.org/licenses/by-nc/4.0/.

\section{ORCID iDs}

Darby W Jack http://orcid.org/0000-0002-9932-0201

Ashlinn K Quinn http://orcid.org/0000-0002-0050-2647

\section{REFERENCES}

1 Murray CJL, Aravkin AY, Zheng P, et al. Global burden of 87 risk factors in 204 countries and territories, 1990-2019: a systematic analysis for the global burden of disease study 2019. The Lancet 2020;396:1223-49.

2 Amegah AK, Quansah R, Jaakkola JJK. Household air pollution from solid fuel use and risk of adverse pregnancy outcomes: a systematic review and meta-analysis of the empirical evidence. PLoS One 2014;9:e113920.

3 Gordon SB, Bruce NG, Grigg J, et al. Respiratory risks from household air pollution in low and middle income countries. Lancet Respir Med 2014;2:823-60.

4 Smith KR, McCracken JP, Weber MW, et al. Effect of reduction in household air pollution on childhood pneumonia in Guatemala (respire): a randomised controlled trial. Lancet 2011;378:1717-26.

5 Mortimer K, Ndamala CB, Naunje AW, et al. A cleaner burning biomass-fuelled cookstove intervention to prevent pneumonia in children under 5 years old in rural Malawi (the cooking and pneumonia study): a cluster randomised controlled trial. Lancet 2017;389:167-75

6 Hartinger SM, Lanata CF, Hattendorf J, et al. Improving household air, drinking water and hygiene in rural Peru: a communityrandomized-controlled trial of an integrated environmental homebased intervention package to improve child health. Int J Epidemiol 2016;45:dyw242-99.

7 Katz J, Tielsch JM, Khatry SK, et al. Impact of improved biomass and liquid petroleum gas stoves on birth outcomes in rural Nepal: results of 2 randomized trials. Glob Health Sci Pract 2020;8:372-82.

8 Kirby MA, Nagel CL, Rosa G, et al. Effects of a large-scale distribution of water filters and natural draft rocket-style cookstoves on diarrhea and acute respiratory infection: a cluster-randomized controlled trial in Western Province, Rwanda. PLoS Med 2019;16:e1002812.

9 World Energy Outlook. Paris, France: international energy agency 2018.

10 lacus SM, King G, Porro G. Causal inference without balance checking: Coarsened exact matching. Political Analysis 2012;20:1-24.
11 Blackwell M, lacus S, King G, et al. Cem: Coarsened exact matching in Stata. Stata J 2009:9:524-46.

12 Jack DW, Asante KP, Wylie BJ, et al. Ghana randomized air pollution and health study (graphs): study protocol for a randomized controlled trial. Trials 2015;16:420.

13 Owusu-Agyei S, Nettey OEA, Zandoh C, et al. Demographic patterns and trends in central Ghana: baseline indicators from the Kintampo health and demographic surveillance system. Glob Health Action 2012;5:19033.

14 Boamah EA, Asante K, Ae-Ngibise K, et al. Gestational age assessment in the Ghana randomized air pollution and health study (graphs): ultrasound capacity building, fetal biometry protocol development, and ongoing quality control. JMIR Res Protoc 2014;3:e77

15 Van Vliet EDS, Asante K, Jack DW, et al. Personal exposures to fine particulate matter and black carbon in households cooking with biomass fuels in rural Ghana. Environ Res 2013;127:40-8.

16 WHO. Handbook: IMCl integrated management of childhood illness. Geneva: World Health Organization, 2005.

17 Mikolajczyk RT, Zhang J, Betran AP, et al. A global reference for fetal-weight and birthweight percentiles. Lancet 2011;377:1855-61.

18 Chillrud SN, Ae-Ngibise KA, Gould CF. The effect of clean cooking interventions on mother and child personal exposure to air pollution: results from the Ghana randomized air pollution and health study (graphs). Journal of Exposure Science \& Environmental Epidemiology 2021:1-16

19 Mishra V, Dai X, Smith KR, et al. Maternal exposure to biomass smoke and reduced birth weight in Zimbabwe. Ann Epidemiol 2004:14:740-7

20 Pope DP, Mishra V, Thompson L, et al. Risk of low birth weight and stillbirth associated with indoor air pollution from solid fuel use in developing countries. Epidemiol Rev 2010;32:70-81.

21 Enwere G, Cheung YB, Zaman SMA, et al. Epidemiology and clinical features of pneumonia according to radiographic findings in Gambian children. Trop Med Int Health 2007;12:1377-85.

22 Zeger SL, Liang KY. Longitudinal data analysis for discrete and continuous outcomes. Biometrics 1986;42:121-30.

23 Thompson LM, Bruce N, Eskenazi B, et al. Impact of reduced maternal exposures to wood smoke from an introduced chimney stove on newborn birth weight in rural Guatemala. Environ Health Perspect 2011;119:1489-94.

24 Alexander DA, Northcross A, Karrison T, et al. Pregnancy outcomes and ethanol cook stove intervention: a randomized-controlled trial in Ibadan, Nigeria. Environ Int 2018;111:152-63.

25 Shen G, Hays MD, Smith KR, et al. Evaluating the performance of household Liquefied petroleum gas Cookstoves. Environ Sci Technol 2018;52:904-15

26 Bruce N, Dora C, Krzyzanowski M. Tackling the health burden from household air pollution (HAP) : development and implementation of new WHO Guidelines. Air Quality and Climate Change 2013;47:32-8.

27 Pope D, Bruce N, Dherani M, et al. Real-life effectiveness of 'improved' stoves and clean fuels in reducing $\mathrm{PM}_{25}$ and $\mathrm{CO}$ : Systematic review and meta-analysis. Environ Int 2017;101:7-18.

28 Burnett RT, Pope CA, Ezzati M, et al. An integrated risk function for estimating the global burden of disease attributable to ambient fine particulate matter exposure. Environ Health Perspect 2014;122:397-403.

29 Ghana Statistical Service. Ghana multiple indicator cluster survey with an enhanced malaria module and biomarker, 2011, final report. Accra, Ghana: Ghana Statistical Service, 2011. https://dhsprogram. com/pubs/pdf/FR262/FR262.pdf 